\title{
FILOLOGIA E CULTURA EPICA: IL CASO DEI TROBADORES
}

\author{
Simone MarCENARO \\ simone.marcenaro@unimol.it \\ Università degli Studi del Molise, Italia
}

Il canzoniere B della lirica galego-portoghese ${ }^{1}$ trasmette al numero 1555 una breve cantiga de escarnio e maldizer del trovatore leonese Fernan Soarez de Quinhones (...1248-1275...):

Lop'Anaia non se vaia, ca, senhor, se s'ora vai e lhi frorecer a faia, a alguen jogará lai.

Se lhi froreç'o bastage, meu senhor, seede sage que prendades dele gage, ca, se s'ora daqui vai, ben fará tan gran domage come Fernan de Romai. Lop'Anaia non se vaia, ca, senhor, se s'ora vai e lhi frorecer a faia, a alguen jogará lai.

Se el algur acha freiras, ou casadas ou solteiras, filha-xas pelas carreiras, e se queren dizer «ai», atá lhis faz as olheiras ben come pres de Cambrai. Lop'Anaia non se vaia, ca, senhor, se s'ora vai e lhi frorecer a faia, a alguen jogará lai.

* Il presente lavoro rientra nelle attività di ricerca connesse al progetto Paleografia, Lingüistica y Filología. Laboratorio on-line de la lírica gallego-portuguesa (FFI2015-68451-P), finanziato dal 'MINECO' spagnolo con fondi provenienti dal FEDER.

${ }^{1}$ Biblioteca Nacional de Lisboa, cód. 10991. 


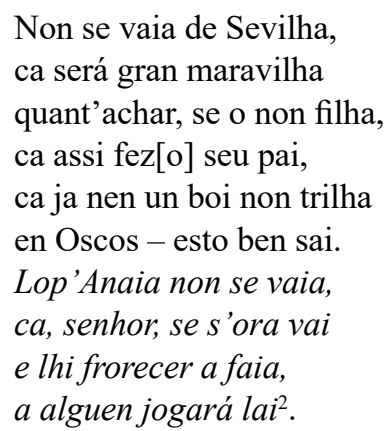

Nella sua edizione, Manuel Rodrigues Lapa sottolineò due elementi importanti: la presenza di gallicismi (soprattutto nei rimanti in -age della prima strofa) e una possibile spia, nella seconda cobla, che il luogo di composizione fosse Siviglia, nella cui campagna di Reconquista (1247-1248) si può supporre che Quinhones abbia partecipato, assieme al fratello Pero Soarez ${ }^{3}$. Quanto ai sintagmi frorecer a faia e frorecer o bastage, il filologo portoghese propone l'interpretazione «se lhe as coisas sairem favoráveis», senza fornire ulteriori spiegazioni. La possibilità di leggere tali espressioni in chiave oscena, favorita soprattutto dal contenuto della seconda cobla, in cui si rende esplicita l'attività che il cavaliere eserciterà verso le freiras, casadas e solteiras, si affaccerà invece più tardi, con l'edizione critica tuttora inedita di João Dionísio (1992)4. In quest'ultima si avanza un'ipotesi che sarà poi seguita da tutti i commentatori posteriori ${ }^{5}$ : la faia e il baston sarebbero traslato per il membro maschile e il verbo frorecer indicherebbe l'erezione. Resta però in ogni caso enigmatica l'origine della metafora evocata da Quinhones, a prescindere dal suo eventuale doppio senso scabroso. L'espressione frorecer a faia

${ }^{2}$ Ed. Manuel Rodrigues Lapa, Cantigas d'escarnho dos cancioneiros medievais galego-portugueses [1965], Vigo, Galaxia, 1970, n. ${ }^{\circ} 143$.

Ho operato minimi ritocchi ai vv. 9-10: nel primo caso si è mutata la punteggiatura proposta da Lapa, nel secondo si è restaurata la forma Fernan de Romai del manoscritto in luogo di Fernand'en Romai di Lapa. Si è inoltre cambiata la punteggiatura dell'edizione Lapa nei vv. $7,8,17,25$ e 27.

${ }^{3}$ Cfr. Carlos Alvar, «Apuntes para una edición de las poesías de Fernan Soárez de Quinhones», in Estudos Portugueses. Homenaje a Luciana Stegagno Picchio, Lisboa, Difel, 1991, pp. 3-14 e António Resende de Oliveira, Depois do espectáculo trovadoresco. A estrutura dos cancioneiros peninsulares e as recolhas dos séculos XIII e XIV, Lisboa, Colibri, 1994, pp. 345-346.

${ }^{4}$ João Dionísio, As cantigas de Fernan Soarez de Quinhones. Edição crítica com introdução, notas e glossário, Faculdade de Letras da Universidade de Lisboa, 1992 (tesi inedita), pp. 161-163. Lo studioso lusitano non si avvede però del significato di faia come metonimia di 'lancia', traducendo invece come 'feito', traslato per «acto de cópula» (p. 163).

${ }^{5} \mathrm{Cfr}$. Xosé Bieito Arias Freixedo, Antoloxía de poesía obscena dos trobadores galego-portugueses, Santiago de Compostela, Plural, 1993, p. 46 (pubblicata anche nella successiva Antoloxía da lírica galego-portuguesa, Vigo, Xerais, 2003, n. $\left.{ }^{\circ} 75\right)$; Graça Videira Lopes, Cantigas de Escárnio e Maldizer dos Trovadores e Jograis Galego-Portugueses, Lisboa, Estampa, 2002, n. ${ }^{\circ} 106$; Simone Marcenaro, $L$ 'equivocatio nella lirica galego-portoghese medievale, Alessandria, Edizioni dell'Orso, 2010, p. 115. 
può infatti tradursi letteralmente come 'fiorire il faggio' e quindi, intendendo faia come metonimia di 'lancia', giacché si parla della raffigurazione di un cavaliere, 'fiorire la lancia'. Una conferma di ciò è data dalla variatio del verso 5 frorecer o bastage, dove bastage è, con ogni evidenza, forma gallicizzante di baston.

Credo che la soluzione vada cercata in quello che si potrebbe definire il retroterra culturale e, più specificamente, letterario del trovatore; un patrimonio che, come si cercherà di dimostrare, ha implicazioni interessanti sia in relazione ad altri autori a lui coevi, sia in rapporto al più ampio -e intrigante- tema degli influssi della letteratura oltrepirenaica in ambito iberico.

Non sfuggirà infatti che l'immagine delle lance fiorite rimanda a un episodio piuttosto famoso della leggenda rolandiana, che troviamo sia nella versione dello Pseudo-Turpino ${ }^{6}$, sia nelle versioni rimate della Chanson de Roland'. Nel libro IV dell'opera latina (capitolo 8) si apprende infatti che Carlo Magno e Milone, in cerca di Agolante per terre iberiche, riescono finalmente a stanare il sovrano moro nella cosiddetta Tierra de Campos, situata vicina al fiume Cea, dove lo stesso Carlo, secondo la leggenda, fondò le chiese di San Facundo e San Primitivo dando così vita alla città leonese di Sahagún. Si conviene dunque che i due eserciti combattano, è il caso di dirlo, ad armi pari, poiché entrambi gli schieramenti si fronteggeranno con cento soldati ciascuno. L'esercito pagano soccombe e, nonostante Agolante aumenti di continuo il numero di guerrieri, non riesce comunque ad avere la meglio. Carlo accorda quindi al sovrano moro di affrontarsi in battaglia aperta e, la sera prima della pugna, avviene il miracolo: i soldati dell'esercito francese piantano le loro lance di fronte alle tende e il giorno seguente quelle di coloro che moriranno in battaglia si ricopriranno di foglie. L'episodio si ripete anche nel cap. $\mathrm{x}$, indicando con chiarezza che le lance fiorite prefigurano la gloria nei cieli dei soldati caduti («qui in bello praesenti accepturi erant martirii palmam pro Christi nomine») ${ }^{8}$; esso verrà poi ripreso nel decimo capitolo della cosiddetta "guida del pellegrino" che si trova nel famoso Codex Calixtinus assieme allo stesso Pseudo-Turpino9 e, come si è detto, verrà richiamato, in forma molto abbreviata, nelle versioni

${ }^{6}$ Cyril Meredith-Jones (ed.), Historia Karoli Magni et Rotholandi ou Chronique du Pseudo-Turpin, Paris, Droz, 1936, p. 110.

${ }^{7}$ L'episodio si ritrova ai vv. 1918-1927 del ms. P e 4652-4671 di L, cfr. Joseph J. Duggan, The song of Roland: the French Corpus, Turnhout, Brepols, 2005, vol. Iv, t. 4, p. 279 e t. 6, p. 113. Uscendo dall'ambito romanzo, il miracolo delle lance si ritroverà poi nella Karlamagnus saga, per la quale si veda Paul Aebischer (ed.), Textes norrois et littérature française du Moyen Âge, Paris, Droz, 1972, p. 135.

${ }^{8}$ Meredith-Jones, Historia Karoli Magni, cit., p. 118.

${ }^{9} \mathrm{Sul}$ rapporto fra le due opere in relazione alla leggenda delle lance fiorite sono ancora validi i rilievi di Joseph Bédier, Les légendes épiques. Recherches sur la formation des chansons de geste, vol. III, Paris, Champion, 1912, pp. 101-102. 
rimate del Roland, dove la fioritura dei mandorli sul campo di battaglia permette a Carlo di distinguere i morti cristiani da quelli saraceni.

L'origine del tema, come ha dimostrato Paolo Cherchi, è da ricercarsi tanto nella matrice classica quanto in quella biblica -la verga di Aronne ${ }^{10}$, oltre a presentarsi, probabilmente come riflesso di tradizioni colte, anche nella cultura folklorica e popolare ${ }^{11}$. Ora, c'è da chiedersi come la leggenda sia arrivata alla cantiga di Fernan Soarez de Quinhones: per farlo, sarà anzitutto conveniente dirigersi verso il resto della produzione in versi del trovatore ${ }^{12}$.

Poco prima del testo già citato, al numero 1553 , il codice $\mathrm{B}$ trasmette un curioso componimento, che vale la pena trascrivere per intero:

\section{Ai, amor, amore de Pero Cantone, que amor tan saboroso e sen tapone!}

Que amor tan viçoso e tan são, queno podesse teer atá o verão! Mais valria que amor de Chorrichão nen de Martin Gonçálvez Zorzelhone. Ai, amor, amore de Pero Cantone, que amor tan saboroso e sen tapone!

Que amor tan delgado e tan frio, mais non creo que dure atá o estio, ca atal era outr'amor de meu tio, que se botou á pouca de sazone. Ai, amor, amore de Pero Cantone, que amor tan saboroso e sen tapone!

Que amor tan pontoso, se cuidades; faze-vos-á chorar, se o gostades, e semelhar-vos-á, se o provades, amor de Don Palaio de Gordone. $A i$, amor, amore de Pero Cantone, que amor tan saboroso e sen tapone!

\footnotetext{
${ }^{10}$ «Quem ex his principes elegero, germinabit virga eius: et cohibebo a me querimonias filiorum Israel, quibus contra vos murmurant. Locutusque est Moyses ad filios Israel: et dederunt ei omnes principes virgas per singulas tribus: fueruntque virgae duodecim absque virga Aaron. Quas cum posuisset Moyses coram Domino in tabernaculo testimonii: sequenti die regressus invenit germinasse virgam Aaron in domo Levi: et turgentibus gemnis eruperant flores, qui, foliis dilatatis, in amygdalas deformati sunt» (Num., cap. XVII, 5-8).

${ }^{11}$ Paolo Cherchi, «'Hastae viruerunt': Pseudo Turpino, Cronaca, capp. VIII e X», Zeitschrift für romanische Philologie, 90 (1974), pp. 229-240.

${ }^{12}$ Non è poi escluso intravedere una convergenza con la tradizione epica spagnola, vale a dire con il Cantar de Mio Cid: il nome Anaia potrebbe infatti richiamare l'appellativo mi anaya, ovverosia 'mio fratello' (anaya proviene dal basco) con il quale il Cid si rivolge ad Alvar Fañez.
} 
Que amor tan astroso e tan delgado; queno tevess'un ano soterrado!

Aquel fora en bõo ponto nado que depois ouvesse del bõa bençone. Ai, amor, amore de Pero Cantone, que amor tan saboroso e sen tapone!

Que amor tan astros'e tan pungente, queno podess'aver en remordente! Mais valria que amor dun meu parente, que mora muit'acerca de Leone.

Ai, amor, amore de Pero Cantone, que amor tan saboroso e sen tapone $!^{13}$

L'interesse di questo testo è molteplice, poiché esso non solo testimonia il gusto di Quinhones verso la forma della ballata con refran iniziale, unita qui alla strofa zagialesca ${ }^{14}$ e già vista nel testo precedente, ma inoltre evidenzia l'uso di rimanti del tutto rari nel corpus dei trobadores, che presentano l'epitesi di -e (o -e paragogica). Analogamente a Lop'Anaia non se vaia, i commentatori che si sono cimentati con la cantiga hanno mostrato un'oggettiva difficoltà nell'interpretare quest'ultimo fenomeno: se Lapa affermava con cautela che poteva trattarsi di una «alteração italianizante dos copistas coloccianos $\rangle^{15}$, i pochi altri pareri al riguardo si limitano a segnalare l'effetto ironico nell'uso di tale ricorso stilistico ${ }^{16}$.

Anche in questa circostanza, invece, sarà utile riferirsi a un modello letterario esterno al dominio lirico, che ancora una volta ricade nell'ambito della chanson de geste. È infatti noto che l'epitesi di $-e$ in rimanti parossitoni è attestata, in ambito iberico, nel frammento del Roncesvalles navarro; il fenomeno, così come studiato da Ramón Menéndez Pidal e, in seguito, da altri studiosi ${ }^{17}$, si affaccia poi con

\footnotetext{
${ }^{13}$ Ed. Lapa, Cantigas d'escarnho, n. ${ }^{\circ} 141$.

${ }^{14}$ Oltre a un'altra cantiga dello stesso Quinhones, LPGP 49,5, il refran iniziale si ritrova nei seguenti testi: LPGP 10,1 (Afonso Soarez Sarraça), 14,13 (Airas Nunez), 18,27 e 18,32 (Alfonso X), 115,5 (Pai Soarez de Taveirós), 147,12 (Roi Paez de Ribela). A questi vanno poi associate le cantigas che presentano una prima strofa, di misura variabile, che possiede schema diverso dal resto delle coblas come LPGP 16,1 di Airas Perez Vuitoron, 18,28 di Alfonso X e il testo attribuito a Roy Martinz do Casal LPGP 145,10 (ma probabilmente spurio e anonimo). Per la diffusione di generi come la ballata o la dansa e il loro rapporto con la lirica mozarabica e le forme francesi di rondeaux e virelai è imprescindibile Vicenç Beltran, «De zéjeles y dansas: orígenes y formación de la estrofa con vuelta», Revista de Filología Española, 84 (1984), pp. 239-266.

${ }^{15}$ Ed. Lapa, Cantigas d'escarnho, cit., n. ${ }^{o} 141$.

${ }^{16}$ Cfr. Dionísio, As cantigas..., cit., pp. 122-124; Videira Lopes, Cantigas d'Escárnio..., cit., n. ${ }^{\circ} 104$.

${ }^{17}$ Cfr. Ramón Menéndez Pidal, La Leyenda de los Infantes de Lara, Madrid, Imprenta de los hijos de José M. Ducazcal, 1896, pp. 418-420; Id., «La forma épica en España y en Francia», Revista de Filología Española, 20 (1933), pp. 345-352; Id., Cantar de Mio Cid, Madrid, Espasa-Calpe, 1946, pp. 1177-1184; Id., Romancero Hispánico, Madrid, Espasa-Calpe, 1953,
} 
regolarità nei più tardi Romances castigliani e, allargando la visuale, si reperisce in una lunga serie di canti popolari che spaziano dalla Castiglia alla Galizia e al Portogallo ${ }^{18}$. Il procedimento ritorna poi nella lirica galego-portoghese in altre tre occasioni, tutte riconducibili al genere della cantiga de amigo (Estevan Coelho, Johan Zorro e Roi Fernandiz de Santiago):

Se oj'o meu amigo soubess', iria migo; eu al rio me vou banhare, al mare. (Estevan Coelho, LPGP 83,3 vv. 1-4) 29,2, vv. 1-4)

El rei de Portugale
barcas mandou lavrare,
e lá irá nas barcas sigo,
mha filha, o voss 'amigo.
(Johan Zorro, LPGP
83,3 vv. 1-4)

Quand'eu vejo las ondas e las muyt'altas ribas, logo mi veen ondas al cor, pola velyda: maldito seja 'l mare que mi faz tanto male. (Roi Fernandiz de Santiago, LPGP 142,20, vv. 1-5)

Non è pacifico chiarire la genesi del fenomeno. Riguardo alla sua apparizione nel latino volgare iberico dei secoli X e XI (si pensi soltanto alla famosa Nota emilianense), le spiegazioni fornite dagli studiosi a partire dal secolo scorso sono disparate, indicando via via la conservazione di elementi arcaizzanti, l'errata latinizzazione di forme volgari per l'influenza analogica di forme in $-a^{19}$ o la semplice tendenza a evitare rime ossitone; ma un dato di fatto assodato, e messo così in luce già chiaramente da Menéndez Pidal, è che nel XIII secolo la $-e$ paragogica era entrata nel volgare castigliano e faceva ormai parte della lingua letteraria, non solo limitata all'epica ${ }^{20}$. L'affermazione di Pidal fu presa però troppo alla lettera da Celso Cunha, il quale, commentando le occorrenze dei tre poeti, pensò che l'epitesi di $-e$ fosse dovuta all'intrinseca struttura ritmica del verso galego-portoghese e che quindi tutti i versi ossitoni -almeno nelle cantigas de amigo- presentassero originariamente paragoge, soltanto in

tomo 1, pp. 119-120; Id., La Chanson de Roland y el neotradicionalismo: orígenes de la épica románica, Madrid, Espasa-Calpe, 1959, pp. 378-382. Per un panorama completo delle diverse posizioni si rimanda a Jacques Horrent, «Les noms Rodlane et Bertlane dans la Nota Emilianense», in Hommage au professeur Maurice Delbouille (Marche romane, num. spécial), 1973, pp. 231-249.

${ }^{18} \mathrm{Si}$ vedano al riguardo i rilievi di Henry R. Lang, «The Spanish estribote, estrambote and related poetic forms», Romania, 45 (1919), pp. 397-421 (specialmente alle pp. 415-421) e Clso Ferreira da Cunha, «Sobre o e paragógico na épica e na lírica», in Id., Lingua e verso, Lisboa, Sá da Costa, 1984, pp. 25-65 (soprattutto alle pp. 46-57).

${ }^{19}$ Quest'ultima ipotesi si ritrova in André Burger, «La question rolandienne. Faits et hypothèses», Cahiers de Civilisation Médiévale, 4 (1961), pp. 269-291.

${ }^{20}$ Menéndez Pidal, La Chanson..., cit., pp. 379-380; l'epitesi si trova anche in testi esterni al genere come la Vida de S. María Egipcíaca. Riguardo invece all'epica, in realtà, l'assenza della - $e$ paragogica nel Cid (ad eccezione di due occorrenze, la prima interpretabile come latinismo, la seconda come semplice errore dello scriba) può far pensare che, a differenza di ciò che afferma Menéndez Pidal, la genesi del fenomeno nell'ambito epico sia più recente $\mathrm{e}$ probabilmente coeva proprio al Roncesvalles (quindi, già in pieno XIII secolo). 
seguito obliterata dai canzonieri colocciani. Quest'ultima ipotesi, va da sé, è totalmente indimostrabile, assai poco verosimile sul piano filologico e, in ultima analisi, da rigettare ${ }^{21}$.

La questione non è di scarsa complessità, ma di sicuro vanno distinti gli affioramenti della $-e$ paragogica nel periodo aurorale della lingua castigliana da quelli della lirica galego-portoghese duecentesca. In quest'ultima, infatti, gli esempi sono, come s'è visto, del tutto sporadici e limitati, il che fa pensare a un ricorso stilistico preciso e non ad un'esigenza generalizzata che si fonda su ragioni metrico-ritmiche. Non va insomma escluso che in queste tre cantigas de amigo la paragoge sia il retaggio di un tono giullaresco, popolareggiante, che ben si comprende per questo genere lirico; non si dimentichi, infatti, che il fenomeno è piuttosto comune nelle kharğat, le quali non possono certo essere tralasciate per la genesi di alcune forme maggiormente popolareggianti della cantiga de amigo, che potrebbero quindi risentire della poesia mozarabica nell'inserzione di tale alterazione metrico-ritmica.

È inoltre necessario differenziare la $-e$ paragogica delle cantigas de amigo da quella usata nelle poesie di Quinhones. In primo luogo, col trovatore leonese ci troviamo nel genere della cantiga satirica, nella quale tutti gli elementi -semantici, lessicali e fonosimboliciconcorrono a costruire l'effetto comico o satirico tipico di questo genere della lirica peninsulare; in altre parole, così come l'immagine tratta dal ciclo rolandiano delle lance fiorite e l'uso di gallicismi venivano utilizzati, tramite la deformazione parodica, per deridere le virtù del cavaliere Lopo Anaia, così qui l'insistenza sui rimanti forniti di $-e$ paragogica vuole chiaramente rimandare a un modello che gli uditori, con ogni probabilità, avevano ben presente. Benché non conosciamo l'identità di nessuno degli individui menzionati da Quinhones, è probabile che questo Pero Canton fosse un cavaliere, o comunque un personaggio di un certo rilievo all'interno del circolo cortese in cui operava Fernan Soarez. E se per canzonare Lopo ci si serviva di allusioni all'epica di matrice oitanica, qui si impiega invece uno stilema direttamente riconducibile all'epica spagnola (o, meglio, a quello che possiamo ricostruirne dai pochi esemplari sopravvissuti).

Ciò che richiama più l'attenzione, pertanto, è la presenza di una pluralità di modelli all'interno di un preciso retroterra cortese. Non sarà allora inutile cercare di ricostruire tanto quei prototipi letterari, quanto il gruppo di poeti che condividevano il medesimo retroterra e, verosimilmente, gli stessi spazi di creazione e esecuzione dei testi che maggiormente evidenziano il riuso degli esemplari gallo-romanzi.

\footnotetext{
${ }^{21}$ Cunha, Sobre o e paragógico..., cit., pp. 60-65. Ad analoghi risultati giunge anche Gladis Massini Cagliari, «A paragoge rítmica na lírica profana galego-portuguesa», in A. C. Macário Lopes - C. Martins (edd.), Actas do XIV Encontro Nacional da Associação Portuguesa de Linguística (Aveiro, 28-30 de Setembro de 1998), Braga, Associação Portuguesa de Linguística, 1999, pp. 169-182.
} 
La conoscenza dell'epica rolandiana in ambito galego-portoghese è cosa nota. Jules Horrent fu il primo a segnalare l'evidente influsso della Chanson de Roland nel curiosissimo (e celebre) testo di Afonso Lopez de Baian, Sediaxi Don Belpelh'en ũa sa maison, che non a caso è indicato nella rubrica dei codici BV come gesta de maldizer. In quell'occasione il nobile portoghese utilizzava la forma della lassa epica monorima, in versi lunghi, enumerando con evidente ironia le caratteristiche dei cavalieri del proprio avversario politico, Men Rodriguez de Briteiros, deriso per la miseria del proprio raffazzonato esercito. A rafforzare l'evidente parodia della chanson de geste, che probabilmente doveva avvenire anche sul piano melodico, alla fine di ogni lassa viene inoltre posto un EOI che richiama chiaramente il celebre $A O I$ del manoscritto oxoniense:

Seiaxi Don Belpelho en ũa sa maison
que chaman Longos, ond'eles todos son.
Per porta lh'entra Martin de Farazon,
escud'a colo en que sev'un capon,
que foi ja poleir'en outra sazon;
caval'agudo que semelha forón,
en cima del un velho selegon,
sen estrebeiras e con roto bardon;
nen porta loriga nen porta lorigon,
nen geolheiras, quaes de ferro son;
mais trax perponto roto sen algodon
e cuberturas dun velho zarelhon;
lança de pinho e de bragal o pendon,
chapel de ferro que xi lhi mui mal pon
e sobarcad'un velho espadarron,
cuitel a cachas, cinta sen farcilhon,
duas esporas destras, ca seestras non son,
maça de fuste que lhi pende do arçon.
a Don Belpelho moveu esta razon:
«iAi, meu senhor!, assi Deus vos perdon,
¿u é Johan Aranha, o vosso companhon
e vosso alferez, que vos ten o pendon?
Se é aqui, saia desta maison,
ca ja os outros todos en Basto son».
EOI!22

La cantiga dimostra quindi che Baian conosceva una versione della Chanson molto prossima a quella anglo-normanna di Oxford, forse già rimata, ma ancora fornita dall'enigmatica formula che, come

${ }^{22}$ Don Afonso Lopez de Baian, Cantigas, Pilar Lorenzo Gradín (ed.), Alessandria, Edizioni dell'Orso, n. ${ }^{\circ}$ VIII, vv. 1-25. 
si sa, non si ritrova in nessun altra versione del Roland. Allo stesso tempo, dovremmo però immaginare che la Chanson fosse conosciuta al pubblico verso il quale Baian si dirigeva: il funzionamento della parodia è infatti strettamente vincolato alla riconoscibilità del testo parodiato, che deve essere ben presente all'uditorio per suscitare l'effetto desiderato (in questo caso, l'attacco a un rappresentante della fazione fedele a Afonso de Boulogne nella guerra civile portoghese del 1246-47) ${ }^{23}$. La conoscenza del Roland fra i trobadores, d'altro canto, è testimoniata da una cantiga di Johan Baveca, malauguratamente frammentaria, che menziona diversi elementi come il passo di Roncisvalle, il non meglio precisato toponimo Poio de Roldam ${ }^{24}$ e l'olifante:

Pero d'Anbroa prometeu de pram que fosse romeu de Sancta Maria, e acabou assy ssa romaria com' acabou a do frume Jordan: ca entonce ata Monpilier chegou e ora per Roçavales passou e tornou-sse do poio de Roldam.<smiles>CCCCCCC1CCCCC1OC(C)C</smiles>

$\mathrm{Ca}$, poys aqui cheguey, ja non diran que non foy ...

$\ldots$

... -am

... en buscar, se non de que podesse poys chufar, e ach' aqui o corno de Rroldam ${ }^{25}$.

\footnotetext{
${ }^{23}$ Se della biografia di Quinhones si è già detto, riguardo a Baian si vedano invece le pp. 7-34 dell'edizione critica curata da Lorenzo Gradín menzionata supra.

${ }^{24}$ Riguardo al toponimo citato da Baveca cfr. Jean-Marie d'Heur, «Roland au Portugal et aussi en Espagne, dans l'art et dans la Littérature», in Hommage au professeur Maurice Delbouille (Marche romane, num. spécial), 1973, pp. 123-146 (cit. alle pp. 142-144). Si veda anche Pilar Lorenzo Gradín, «Modelos épicos y artúricos en la lírica gallego-portuguesa», in Marco Piccat e Lura Ramello (ed.), Epica e cavalleria nel medioevo. Atti del Seminario Internazionale, Torino, 18-20 novembre 2009, Alessandria, Edizioni dell'Orso, 2011, pp. 77-97.

${ }^{25}$ Ed. Johan Baveca, Poesie, Carmelo Zilli (ed.), Bari, Adriatica, 1977, n. ${ }^{\circ} 25$.
} 
In questo caso, l'accenno alla toponimia rolandiana è funzionale a un tema molto frequentato dai trovatori galego-portoghesi, quello del viaggio verso Oltremare per il quale il trovatore Pero de Ambroa è bersagliato in diverse cantigas burlesche ${ }^{26}$. Benché non sia necessario ipotizzare per forza l'esistenza di un testo concreto alla base delle citazioni di Baveca, bisogna ricostruire il possibile grado di penetrazione dell'epica rolandiana negli ambienti trobadorici per comprendere la fisionomia dei possibili ipotesti e sono le stesse cantigas a fornirci spunti ulteriori.

Esistono infatti altri elementi che ci possono aiutare a ricostruire l'ambiente in cui la rielaborazione del materiale epico serviva a consolidare l'effetto satirico delle cantigas de escarnio. E uno di questi ci riconduce di nuovo a Fernan Soarez de Quinhones, il quale, nella seguente cantiga, richiama con ogni evidenza la gesta satirica di Afonso Lopez de Baian:

\author{
Contar-vos-ei costumes e feituras \\ dun cavalo que traj'un infançon: \\ á pees moles e as sedas duras \\ e tem'o freo e esporas non; \\ é velh'e sesgo nas aguilhaduras; \\ e non encalçaria un leiton, \\ e encalçaria mil ferraduras.
}

De dia empeça ben: com'a escuras, non s'alevant'ergo su o bardon; non corre senon pelas mataduras,

${ }^{26}$ In realtà Pero de Ambroa intraprese davvero il viaggio in Terrasanta, se si tiene fede a un documento proveniente da un monastero di Ferreira de Pallares, nella provincia di Lugo (cfr. José Antonio Souto Cabo, «Pero Garcia d'Ambroa e Pero de Ambroa», Revista de Literatura Medieval, 18 (2006), pp. 225-248, a p. 234). Il tema di Ultramar costituisce un vero e proprio ciclo nelle cantigas satiriche peninsulari, a sua volta legato al nutrito gruppo di composizioni che deridono la soldadeira Maria Pérez Balteira, anch'essa schernita in ragione della sua pretesa di farsi crociata. Si leggano a tal proposito Cfr. Carolina Michaëlis de Vasconcelos, «Randglossen zum altportugiesischen Liederbuch. VII. Eine Jerusalempilgerin und andre Kreuzfahrer», Zeitschrift für romanische Philologie, 25 (1901), pp. 533-560; Carlos Alvar, «La cruzada de Jaén y la poesía gallego-portuguesa», in Actas del I Congreso de la Asociación Hispánica de Literatura Medieval, Santiago de Compostela, de 2 a 6 diciembre de 1985, Barcelona, Promociones y Publicaciones Universitarias, 1988, pp. 139-144; M. Cleofé Tato García, «Las cantigas de ultramar gallego-portuguesas», in D. Kremer (ed.), Actes du XVIII Congrès International de Linguistique et de Philologie Romanes, Université de Trèves (Trier) 1986, Tübingen, Niemeyer, 1988, t. 6, pp. 190-201; Gerardo Pérez Barcala e M. ${ }^{a}$ del Carmen Rodríguez Castaño, «A viaxe paródica a Ultramar: da canción de cruzada á cantiga de escarnio», in Elvira Fidalgo Francisco e Pilar Lorenzo Gradín (eds.), Estudios galegos en homenaxe ó Profesor Giuseppe Tavani, Santiago de Compostela, Xunta de Galicia, 1994, pp. 135-151; Giuseppe Tavani, «A viagem ao ultramar nas cantigas medievais», in S. Serafin (ed.), Un lume nella notte. Studi di iberistica che allievi ed amici dedicano a Giuseppe Bellini, Roma, Bulzoni, 1997, pp. 379-387; Esther Corral Díaz, «María Balteira e a peregrinación a Terra Santa», in C. A. González Paz (ed.), Mujeres y peregrinación en la Galicia Medieval, Santiago de Compostela, Instituto de Estudios Gallegos 'Padre Sarmiento' CSIC, 2010, pp. 81-97. 
nen traz caal, se enas unhas non, u trage mais de cen canterladuras; e as sas rẽes sempre magras son; mais nas queixadas á fortes grossuras.

E quando lhi deitan as armaduras, logu'el faz contenente de foron; e, se move, tremen-lh'as comas duras, come doente de longa sazon. Á muit'espessas as augaduras, e usa mal, se nos geolhos non, en que trage grandes esfoladuras.

Non vos contarei mais eu sas feituras; mais, com'eu creo no meu coraçon, quen x'en gran guerra andass'a loucuras, en feúza daqueste cavalon, falecer-lh'-ia el nas queixaduras; e, ena paz, non ar sei eu cochon que o quisesse trager nas Esturas ${ }^{27}$.

La ripresa è palese sia a livello tematico, siccome entrambi i testi condividono il medesimo tema del cavaliere miserabile, sia a livello formale, poiché si verificano recuperi puntuali dalla gesta. Ciò avviene mediante rapporti interdiscorsivi -la descrizione disfemica della cavalcatura presente nella seconda "lassa" del testo di Baian-e intertestuali, questi ultimi assicurati dai rimanti comuni bardon, son, foron, sazon.

Non è facile stabilire quale sia la direzione dello scambio poetico. Sappiamo però che Baveca e Baian parteciparono alla riconquista di Siviglia, terminata nel novembre 1248 , e potrebbe essere questa l'occasione in cui essi vennero a contatto. Il testo di Baian non è databile con precisione, ma è verosimile pensare al periodo immediatamente successivo alla presa di potere di re Afonso $\mathrm{II}^{28}$, avvenuta nel gennaio 1248; sappiamo poi che Baian non dovette trattenersi presso la corte castigliana oltre il 1250 e che tornò in Portogallo prima dell'incoronazione del futuro Alfonso X di Castiglia (1252) ${ }^{29}$. Riguardo al testo di Johan Baveca, è decisivo capire a quale crociata si riferiscano le cantigas che menzionano la spedizione in Terrasanta di Pero de Ambroa. Sono state proposte a tal riguardo diverse ipotesi ${ }^{30}$, ma la

${ }^{27}$ Ed. Lapa, Cantigas d'escarnho, n. ${ }^{\circ} 144$

${ }^{28}$ Don Afonso de Baian, Cantigas, cit., pp. 181-182.

${ }^{29}$ Cfr. Resende, Depois o espectáculo, cit., p. 308.

${ }^{30} \mathrm{Si}$ consulti a tal proposito la bibliografia fornita da Esther Corral Díaz, «La peregrinación como contratexto en la lírica gallego-portuguesa», in Ead. (ed.), In marsupiis peregrinorum. Circulación de textos y imágenes alrededor del Camino de Santiago de la Edad Media, Firenze, Edizioni del Galluzzo per la Fondazione Franceschini, 2010, pp. 365-384. 
più assennata sembra essere quella della settima crociata, la prima delle due promosse da Luigi IX, effettuata fra 1248 e $1254^{31}$; se si ammette questa data, allora, anche il testo di Baveca potrebbe essere compreso nel periodo che precede l'incoronazione del Rey Sabio, e più precisamente fra gli ultimi anni del quarto decennio del Duecento e i primi della decade successiva.

Considerando l'impatto che la parodia di Baian della Chanson de Roland dovette avere fra i trobadores, è forse più verosimile pensare al suo influsso diretto giocato su Contar-vos-ei di Quinhones, che ebbe modo di riprendere alcuni elementi del testo in un lasso di tempo molto breve, nel cenacolo dei trovatori raccolti attorno alla figura di Alfonso infante di Castiglia. Nel medesimo gruppo, come desumiamo dalle allusioni della sua cantiga frammentaria, si trovava forse anche Johan Baveca -sicuramente a Siviglia nel 1248-, che partecipò a questo processo di ripresa burlesca dell'epica rolandiana con il testo di cui oggi conserviamo soltanto due strofe ${ }^{32}$.

A questo punto, vi sono elementi sufficienti per ricostruire lo spazio cortese in cui operavano Quinhones e i suoi sodali. Negli anni 1247-48, durante la presa di Siviglia, alla corte dell'infante Alfonso erano compresenti tre poeti che utilizzano immagini e stilemi della produzione epica per le loro cantigas satiriche. Possiamo supporre, quindi, che in tale ambiente fossero penetrati testi di matrice rolandiana rielaborati dai trobadores, consci del fatto che questi dovevano essere già noti al proprio pubblico. Resta però da comprendere qualcosa di più sulla reale conformazione di questi intertesti. Se per Baian, come si è visto, ci troviamo di fronte a una chanson de geste forse rimata, ma che di sicuro conservava il tratto arcaico della misteriosa invocazione che caratterizza il Roland oxoniense, su Quinhones agiscono invece almeno due modelli differenti: da una parte, l'episodio delle lance fiorite, che potrebbe aver ripreso tanto dallo Pseudo-Turpino quanto da versioni simili a quelle rimate del Roland (e attraverso l'uso, non lo si dimentichi, di numerosi gallicismi); dall'altra, un cantar forse simile al Roncesvalles, nel quale si ritrovava la -e paragogica.

Come questi testi fossero fruiti, assimilati e in seguito rielaborati dai trovatori non è semplice immaginare. Non sarà però inutile ricordare due passi tratti dal De Rebus Hispaniae di Rodrigo Jiménez de Rada (terminato nel 1243) e della Primera Crónica General (o Estoria de España), entrambi rivolti a descrivere l'espansione della leggenda rolandiana in ambito iberico:

\footnotetext{
${ }^{31}$ Si veda al riguardo Alvar, «La cruzada de Jaén», cit.

32 Anche Quinhones godette di una certa fortuna fra i trovatori iberici, come provano le riprese dei rimanti faia, vai, ai e del sintagma pres de Cambrai in un testo di Johan Airas de Santiago (LPGP 63,15), operante fra la seconda metà del Duecento e i primi anni del secolo successivo.
} 
Nonnulli histrionum fabulis inhaerentes ferunt Carolum ciuitates plurimas, castra et oppida in Hispaniis acquisisse, multaque praelia cum Arabibus strenue perpetrasse, et stratam publicam a Gallis et Germania ad sanctum Iacobum recte itinere direxisse... (De rebus Hispaniae)

Et algunos dizen en sus cantares et en sus fablas de gesta que conquirio Carlos en Espanna muchas çipdades et muchos castiellos, et que ouo y muchas lides con moros, et que desenbargo et abrio el camino desde Alemannia fasta Sanctiago. (Primera Crónica General) ${ }^{33}$

I due brani forniscono notizie piuttosto precise. Da un lato, infatti, ci informano della presenza di giullari presso le corti castigliane, $i$ quali, dovremmo immaginare, avevano materiale rolandiano nel proprio repertorio di cantares che traducevano o adattavano; dall'altro, si desume che questo materiale dovesse coincidere con le versioni rimate del Roland, poiché sono le uniche che introducono il tema della liberazione della Spagna fino a Santiago de Compostela. Ora, non si deve per forza pensare che la conoscenza delle chansons de geste attraverso gli adattamenti in lingua castigliana dovesse provenire da un singolo canale di diffusione. È però altrettanto vero che il modo più efficace e immediato per la penetrazione della leggenda di Carlo Magno e Rolando fosse rappresentato proprio quegli histriones che le due cronache menzionano esplicitamente; essi garantivano infatti la propagazione capillare a un pubblico piuttosto vasto, nel quale erano sicuramente compresi anche alcuni trobadores.

I dati che provengono dalle cronache elaborate presso la corte di Alfonso X e Sancho IV, d'altra parte, descrivono piuttosto bene il modo in cui le officine scrittorie si approvvigionavano dei materiali narrativamente vicini alle chansons de geste, tanto di origine oitanica quanto autoctona. Sono stati infatti sottolineati a più riprese ${ }^{34} \mathrm{i}$ passi in cui, analogamente alle due citazioni appena riportate, i giullari vengono chiamati esplicitamente in causa, spesso per contestare gli avvenimenti che essi cantavano con la più concreta realtà delle fonti storiche. Nelle crónicas si veniva così a creare un preciso dualismo fra ciò che proveniva dalla fruizione orale di gesta epiche, che dobbiamo pensare frequenti nell'epoca di cui stiamo parlando, e le fonti di natura essenzialmente cronachistica -e quindi scritte- che gli estensori della storiografia ufficiale talora reputavano maggiormente fededegne. Da questo dualismo si evince allora che l'acquisizione dell'epica francese dovesse prodursi, nell'ambiente della Castiglia

\footnotetext{
${ }^{33}$ Entrambi i passi sono citati da Theodor Heinermann, Untersuchungen zur Entstehung der Sage von Bernardo del Carpio, Halle a. S., Niemeyer, 1927, p. 13.

${ }^{34}$ Per un bilancio della questione si veda Diego Catalán, La épica española: nueva documentación y nueva evaluación, Madrid, Fundación Ramon Menéndez Pidal, Seminario Menéndez Pidal, Universidad Complutense de Madrid, 2000, pp. 13-15.
} 
duecentesca, per via soprattutto orale, diffusa da jograres che conoscevano ormai un repertorio stabile di cantares o in lingua d'oïl, o tradotte in castigliano, talvolta conservando elementi notevolmente arcaici (l'EOI di Baian ne è testimone). È allora facile immaginare che il giovane Alfonso e i suoi sodali si trovassero in un ambiente cortese tutto sommato provvisorio, legato alla contingenza della campagna sivigliana, e che la conoscenza delle canzoni di gesta fosse molto più facilmente d'occasione, avvenuta quindi per udita e non attraverso la lettura.

Il fatto che la cantiga su Lopo Anaia di Quinhones e la gesta parodica di Baian esibiscano gallicismi non dovrà essere interpretato come tentativo mimetico nei confronti dell'antico francese, bensì come marca burlesca che dimostra con chiarezza quale fosse, pur in epoca così avanzata, la matrice del testo parodiato: indicandone così non la provenienza linguistica, ma piuttosto, per così dire, l'appartenenza culturale. E questo è un elemento cruciale per capire qualcosa di più sul ruolo di Alfonso $\mathrm{X}$ nel processo di rielaborazione della cultura letteraria francese, ruolo che forse, ad oggi, non è ancora stato stabilito con sufficiente esaustività. Evidentemente, fra la quarta e la quinta decade del Duecento alla corte dell'infante Alfonso il riferimento principale per il genere epico era ancora la chanson de geste oitanica, nonostante l'epica castigliana fosse ormai già conosciuta $\mathrm{e}$ diffusa nella penisola iberica. Una volta incoronato, attorno al 1270, sappiamo però che Alfonso promosse la redazione della Primera Crónica General, ampliata e terminata sotto Sancho IV nel 1289, per la quale egli fece raccogliere una notevole messe di fonti scritte. Tra queste fonti dovevano figurare, com'è ovvio, anche opere di argomento epico, viste le ben note prosificazioni del Cid e di perduti cantari di gesta come quello di Bernardo del Carpio, di Fernán Gonzalez o degli Infantes de Lara; per questo motivo, si è giustamente osservato come le leggende epiche rifuse nella storiografia alfonsina restituiscano un'immagine non troppo favorevole alla cultura francese e orientata verso una sorta di "nazionalismo" epico ${ }^{35}$. L'attenzione filo-ispanica, massima nella citazione del Mio Cid, è certo comprensibile nell'ottica di una storiografia nazionale, tanto più se la materia epica serviva a costruire un discorso di tipo, appunto, storico-cronachistico. Ma è senz'altro curioso notare come, nello spazio di circa vent'anni, al giovane Alfonso ancora imbevuto di letteratura d'oïl si sostituisca il monarca attento a costruire una narrazione storiografica che doveva per forza prescindere dalla materia oitanica, più facilmente vincolata a una visione del mondo poco consona alla nuova ideologia della corte castigliana.

${ }^{35}$ Per cui si rimanda a Maria Luisa Meneghetti, «Almanzor, Çorraquin Sancho e i primi passi dell'epica romanza», Medioevo Romanzo, 22 (1998), pp. 313-325. 
Dovremo allora pensare che il passaggio dalla giovinezza alla reggenza del regno coincida proprio con l'abbandono, sancito da un monarca ormai proiettato verso aspirazioni imperiali, di quella letteratura che probabilmente lo aveva appassionato, assieme ai trovatori di cui si era circondato, negli anni delle campagne militari promosse dal padre Fernando III. Una letteratura che, grazie ai testimoni dei trobadores, mostra ancora la sua vitalità a cavallo della metà del secolo, in quella Spagna divenuta uno tra i più importanti crocevia per la circolazione europea del materiale epico: capace, da una parte, di dare linfa vitale alla materia di Francia con opere come il Roncesvalles (forse composto negli stessi anni delle cantigas di Quinhones, Baian e Baveca) ${ }^{36}$, e, dall'altro, sedotta dalle narrazioni di eroi nazionali come Rodrigo Díaz de Vivar o Bernardo del Carpio, trasmesse dalle esecuzioni dei giullari fino alle grandi cronache storiche.

Recibido: 16/05/2017

Aceptado: 10/08/2017

${ }^{36}$ Sussistono due ipotesi per la datazione del frammento navarro: la prima di Menéndez Pidal, che pensa al primo terzo del Duecento, mentre la seconda, di Jules Horrent, propende per la fine del XIII secolo (cfr. R. Menéndez Pidal, «Roncesvalles: un nuevo cantar de gesta español del siglo XIII», Revista de Filología Española, 4 (1917), pp. 105-214 e Jules Horrent, "Roncesvalles», étude sur le fragment de cantar de gesta conservé à l'Archivo de Navarra (Pampelune), Paris, Belles Lettres, 1951). 


\title{
$\cos$
}

\section{FILOLOGIA E CULTURA EPICA: IL CASO DEI TROBADORES}

\begin{abstract}
RESUMEN: Alcune cantigas de escarnio e maldizer del trovatore galego-portoghese Fernan Soarez de Quinhones alludono, in forma parodica e burlesca, alla letteratura epica, sia attraverso l'uso di forme e stilemi propri del genere, sia attraverso una rete di rimandi intertestuali. L'analisi e lo studio dei possibili intertesti utilizzati dal trovatore per le sue canzoni satiriche possono dirci qualcosa di più in merito alla cultura letteraria del circolo di autori che si riunirono alla corte del futuro Alfonso X, ancora infante, nel quarto decennio del XIII secolo; allo stesso tempo, sarà possibile fornire dati utili sull'influsso dell'epica antico-francese nell'Iberia medievale.
\end{abstract}

PAROLE-CHIAVE: Trovatori galego-portoghesi, cantigas de escarnio e maldizer, Chanson de geste, Alfonso X di Castiglia.

\section{PHILOLOGY AND EPIC CULTURE: THE CASE OF THE TROBADORES}

\begin{abstract}
Some cantigas de escarnio e maldizer by the Galician-Portuguese Troubadour Fernan Soarez de Quinhones hint at epic Literature in a parodic and satirical manner, through the use of forms and style of the epic genre but also thanks to a deep network of intertextual relationships. The study and analysis of the possible sources used by the author in his satirical poems can tell us something more about the literary culture of the Troubadours gathered at the court of the infante Alfonso, in the fourth decade of the $13^{\text {th }}$ century; at the same time, it will be possible to provide useful data on the influence of Old French epic poetry in medieval Iberia.
\end{abstract}

Keywords: Galician-Portuguese Troubadours, cantigas de escarnio e maldizer, Chanson de geste, Alfonso X of Castile. 